\title{
Improved dose-calculation accuracy in proton treatment planning using a simplified Monte Carlo method verified with three-dimensional measurements in an anthropomorphic phantom
}

\author{
Kenji Hotta $^{1}$, Ryosuke Kohno ${ }^{2}$, Yoshihisa Takada ${ }^{1}$, Yousuke \\ Hara $^{1}$, Ryohei Tansho ${ }^{1}$, Takeshi Himukai ${ }^{3}$, Satoru Kameoka ${ }^{2}$, \\ Taeko Matsuura ${ }^{2}$, Teiji Nishio ${ }^{2}$, and Takashi Ogino ${ }^{2}$ \\ ${ }^{1}$ Institute of Applied Physics, University of Tsukuba, 1-1-1 Tennoudai, Tsukuba, \\ Ibaraki 305-8573, Japan \\ ${ }^{2}$ National Cancer Center Hospital East, 6-5-1, Kashiwanoha, Kashiwa, Chiba \\ 277-8577, Japan \\ ${ }^{3}$ National Institute of Radiological Sciences, 4-9-1, Anagawa, Inage, Chiba, 263-8555, \\ Japan \\ E-mail: y-takada@bk.tsukuba.ac.jp
}

\begin{abstract}
Treatment planning for proton tumor therapy requires a fast and accurate dose calculation method. We have implemented the Simplified Monte Carlo (SMC) method in the treatment planning system of the National Cancer Center Hospital East for the double-scattering beam delivery scheme. The SMC method takes into account the scattering effect in materials more accurately than the pencil beam algorithm by tracking individual proton paths. We confirmed that the SMC method reproduced measured dose distributions in a heterogeneous slab phantom better than the pencil beam method. When applied to a complex anthropomorphic phantom, the SMC method reproduced the measured dose distribution well, satisfying an accuracy tolerance of $3 \mathrm{~mm}$ and $3 \%$ in the gamma index analysis. The SMC method required approximately 30 minutes to complete the calculation over a target volume of 500 cc, much less than the time required for the full Monte Carlo calculation. The SMC method can be a candidate of practical calculation technique with sufficient accuracy for clinical application.
\end{abstract}

PACS numbers: 87.55.D-, 87.55.K-

Submitted to: Phys. Med. Biol. 


\section{Introduction}

In proton tumor therapy, tumor control while sparing adjacent organs requires a good treatment plan to maximize dose delivery to the target volume. The optimum plan is formulated by evaluating the calculation results for a variety of beam configurations. The dose calculation method must be fast and accurate.

Many facilities currently use pencil beam algorithms (PBAs) (Petti 1992, Hong 1996, Szymanowski 2001) for treatment planning. The required calculation time is relatively short and the accuracy of these algorithms is reasonable when the tumor is surrounded by structures of intermediate complexity. PBAs express the dose distribution formed by a mono-energetic proton pencil beam as a product of the depthdose distribution in water obtained from measurements or Monte-Carlo calculations and an off-axis radial function defined as a two-dimensional Gaussian function with an rms value determined by scattering in the materials along its central axis. The dose distributions of multiple pencil beams at various incident positions and energies are summed to obtain the dose distribution in the patient. Kohno et al developed a RangeModulated-Pencil-Beam Algorithm (RMPBA) to shorten the calculation time while maintaining accuracy by using a measured depth-dose distribution for the combined beam rather than summing the contribution of protons at each specific energy (Kohno 2001).

Though PBAs perform well for homogeneous targets, the accuracy is decreased in targets with large lateral heterogeneity. Since the PBAs assume that the central axis is a straight line and determine the energy deposit and the lateral spread due to materials along the central axis, they do not include the effects of lateral density heterogeneity on the dose distribution. The PBAs also use a zero-thickness collimator approximation ignoring the edge scattering in the aperture collimator. These limitations decrease the dose-calculation accuracy of PBAs in heterogeneous media. In order to improve accuracy, Kanematsu et al developed a PBA variant that subdivides the pencil beam kernels into sub-pencil beams when it encounters a large heterogeneity (Kanematsu 2009).

The clinical application of full Monte Carlo calculations such as MCNPX (Waters 2002) or Geant4 (Agostinelli 2003) has been investigated (Paganetti 2008). Although they are capable of more accurately computing dose distribution, they require a long calculation time, up to 6 hours per patient even using more powerful cluster machine than ours (Paganetti 2008). To reduce the calculation time, fast pseudo-Monte-Carlo algorithms were proposed ( $\mathrm{Li} 2005$, Yepes 2009).

Sakae et al (2000) developed a Simplified Monte Carlo (SMC) method to obtain fast and accurate dose calculation in heterogeneous targets, and the accuracy of the method in simple targets was verified by Kohno et al (Kohno 2002, 2003). Since the SMC method tracks individual particles, it includes lateral density heterogeneity effects on the dose distribution. A second advantage of the SMC method is easy implementation since it can use same input data for PBAs. 
We implemented the SMC method in the clinical treatment planning system of the National Cancer Center Hospital East (NCCHE, Japan). We demonstrated the effectiveness of the SMC method by comparing the calculation results with measurement results in a heterogeneous slab phantom, and that in an anthropomorphic phantom simulating the complexity encountered in a clinical situation. The SMC results were also compared to RMPBA calculations. The data were analyzed using a number of methods, including a variant of the $\gamma$-index method (Low 1998) with an accuracy tolerance of 3 $\mathrm{mm}$ and $3 \%$.

\section{Materials and Methods}

\subsection{Calculation Model}

A right-handed Cartesian system was used for dose calculation coordinates in which the central beam axis coincided with the z-axis and the gantry rotated about the $\mathrm{y}$ axis. Target data in the original CT coordinate system were transformed into the dose-calculation coordinate system using the gantry and couch rotation angles.

The SMC method begins tracking individual protons at the entrance to the range compensator $(\mathrm{RC})$. The initial beam parameters were provided by the effective-source model with the model parameters determined by measurements (Hong 1996, Symanovski 2001). The model provides the standard deviation of the initial angular distribution at any point on the entrance plane. The proton fluence distribution was based on the lateral dose distribution measured without the $\mathrm{RC}$ and aperture collimator. In the system arrangement at the NCCHE, the RC is placed upstream of a patient-aperture collimator. For calculation of range loss and scattering of individual protons in material, polyethylene $\mathrm{RC}$ and $60 \mathrm{~mm}$-thick brass aperture collimator were divided into segments with a thickness of $1 \mathrm{~mm}$ along z-axis. Patient volume was divided into cubic voxels with twice the edge length of the CT pixels. Each particle was characterized in terms of position, the direction expressed by the two projection angles, and the residual range in water. The trajectory of each particle was tracked by assuming multiple Coulomb scattering with scattered projection angles expressed as a normal random number with a standard deviation calculated using the Highland formula (Highland 1975, Highland 1979). The energy loss of a proton in a segment of material was calculated using the water equivalent model (Chen 1979). We assumed that the relative dose deposit in a patient voxel could be obtained from the measured depth-dose distribution in water. One reason for the shorter calculation time of the SMC method compared with full Monte Carlo methods is the simplification in which the dose deposit in materials is calculated using the measured depth-dose distribution for a mono-energetic proton beam in water, and ignoring absorption and lateral scattering due to nuclear reaction. Note that use of the measured depth-dose curve in water implicitly includes some averaged effects from nuclear interactions.

The calculation method was compared to the RMPBA with the measured effective 
source model.

\subsection{Experiment}

We verified the calculation accuracy of the SMC method by comparing the calculation results with the measured dose distributions in the heterogeneous phantoms described in sections 2.2.1 and 2.2.2. The proton beam was extracted from the $235-\mathrm{MeV}$ cyclotron at the NCCHE. For some experiments the energy was reduced using the energy-selectionsystem (ESS), and the beam was transferred to a passive beam spreading system using the double-scattering method (Nishio 1999, Tachikawa 1999).

A PTW 2D Array seven $29^{\mathrm{TM}}$ was used for dose detection. This is a two-dimensional detector matrix containing 729 ionization chambers in a 10 mm-pitch $27 \times 27$ array developed by (CPTW Freiburg GmbH. Spezi reported the successful application of this detector to radiation therapy and verified the performance (Spezi 2005). The sensitive volume of a unit chamber is $5 \mathrm{~mm} \times 5 \mathrm{~mm} \times 5 \mathrm{~mm}$. The ionization chambers of the array are open to the air. The offset thickness from the entrance surface to the center of the sensitive volume is $8 \mathrm{~mm}$ in WEL.

To compare the calculation results and measurements under the same conditions, we corrected the calculation depths by the offset thickness when calculating the dose distributions. We also convolved the calculation results with the detector cell size of 5 $\mathrm{mm} \times 5 \mathrm{~mm}$.

2.2.1. Slab phantom The heterogeneous slab phantom depicted in figure 1 was used for evaluation of the SMC method. We investigated lateral density heterogeneity effects on dose distribution using a $150 \mathrm{-MeV}$ proton beam passing through a ridge filter with a Spread-Out Bragg Peak (SOBP) width of $80 \mathrm{~mm}$. The phantom was constructed by combining $10 \mathrm{~mm}$-thick component slabs made from Tough Water (TW), Tough Lung (TL), and Tough Bone (TB) (C)Kyoto Kagaku Co., Ltd). The water-equivalentthickness ratios (Takada 2008) of TW, TL, and TB are 1.01, 0.34, and 1.40. We designed the $\mathrm{RC}$ and the aperture collimator for the cube-shaped target with a volume of $800 \mathrm{cc}$ (indicated by the red line in figure 1).

Lateral dose distributions were measured at depth of $\mathrm{z}=0 \mathrm{~mm}, 20 \mathrm{~mm}, 40 \mathrm{~mm}, 60$ $\mathrm{mm}, 80 \mathrm{~mm}$, and $90 \mathrm{~mm}$. A stack of the phantom slabs was mounted on the detector to measure the dose distribution in each depth as shown in the figure 1 . When a different phantom stack was mounted, we fixed the distance between the aperture collimator and the phantom entrance surface by adjusting the vertical position of the patient couch. Since the chamber pitch was $10 \mathrm{~mm}$, we shifted the detector by $5 \mathrm{~mm}$ in the $\mathrm{x}$ and $\mathrm{y}$ directions to obtain measurements with a lateral sampling pitch of $5 \mathrm{~mm}$. Each measurement was repeated three times at each depth and averaged to obtain the lateral dose distribution.

The simulation required approximately 40 minutes with a target voxel size of $1 \mathrm{~mm}$ per side using $1.14 \times 10^{8}$ generated protons on 4 cores (two dual-core $2.4 \mathrm{GHz} \mathrm{AMD}$ 
Opteron CPUs, 4 jobs running in parallel). All the dose data sets were normalized at a point $(\mathrm{x}=30 \mathrm{~mm}, \mathrm{y}=60 \mathrm{~mm})$ in a flat dose region for comparison of measurements and calculations. The estimated mean statistical error of the calculated dose in the target region was $1 \% \mathrm{rms}$, and that of the convolved calculated dose was $0.2 \% \mathrm{rms}$. Reduction of the error in the convolved calculated dose came from the larger voxel size.

2.2.2. RANDO phantom We used the head portion from a RANDO@ phantom produced by the (C) Phantom Laboratory to simulate the complex arrangement of materials experienced in clinical applications. The RANDO phantom mimics the density distribution in the human head using resins with various compositions. Figure 2 contains the median sagittal and median horizontal CT images and the PTV. The phantom is composed of horizontal layers $25 \mathrm{~mm}$ thick. We measured the dose distributions in the layers with the z-index numbered from 1 to 7 as shown in the figure. To simulate clinical situations, we followed the actual patient treatment procedure: obtaining a CT, delineating the PTV, determining the beam direction, manufacturing the corresponding $\mathrm{RC}$ and aperture collimator, aligning the reference surface markers on the phantom with laser cross-hairs, and irradiating the phantom on the patient couch. We designed a treatment plan assuming a head and neck cancer with a volume of approximately 500 cc. Smearing distance of the RC (Kooy 2008) was taken at $4.5 \mathrm{~mm}$. Since the measurement plane was limited to the horizontal plane due to the layered structure of the phantom, the irradiation direction was also limited to downward from the top of the head. Although the results have no clinical significance due to the unrealistic selection of the irradiation direction, it simulates the dose distribution in the complex heterogeneous region typically found in the head and neck cases.

We used a $235-\mathrm{MeV}$ proton beam with a SOBP width of $80 \mathrm{~mm}$. We mounted a stack of phantom layers on the detector to measure the dose distribution in each measurement plane. The distance between the aperture collimator and the phantom entrance surface was fixed by adjusting the couch height, again. The reported results are the average of three measurements. To estimate the effect of set-up errors on the dose distribution, we repeated the set-up and measurement procedure three times on the $\mathrm{z}=4$ layer where a complicated lateral dose distribution was expected.

The simulation required approximately 30 minutes when the target voxel size was $1.17 \mathrm{~mm}$ on a side and the number of generated particles was $4.68 \times 10^{7}$ on 4 cores (two dual-core $2.4 \mathrm{GHz}$ AMD Opteron CPUs, 4 jobs running in parallel). All the dose data sets were normalized with reference to the dose at the iso-center for comparison. The estimated mean statistical error of the calculated dose in the target region was $1.2 \%$ $\mathrm{rms}$, and that of the convolved calculated dose was $0.25 \% \mathrm{rms}$. 


\section{Results}

\subsection{Slab phantom}

Figure 3 compares the iso-dose distributions obtained from (a) measurements, (b) the SMC calculation, and (c) the RMPBA calculation. In the figure, we notice that the high-dose region around $\mathrm{x}=0 \mathrm{~mm}$ extends to a region deeper than $50 \mathrm{~mm}$ only in the RMPBA. Such differences of dose distribution are shown more clearly in lateraland depth-dose profiles of figure 4. The SMC method reproduced the measurement results better than the RMPBA in three regions. The first is the peripheral high-dose region around $\mathrm{x}= \pm 50 \mathrm{~mm}$ in figure 4 (a) that is influenced by the scattered and energydegraded protons interacting with the edge of the aperture collimator. The SMC method accurately reproduced the measured dose distribution, while the RMPBA does not take into account edge-scattered proton paths and cannot reproduce the dose distribution well in this region. The second region is a dose reduction at depths between $\mathrm{z}=40$ $\mathrm{mm}$ and $\mathrm{z}=70 \mathrm{~mm}$ in the vicinity of $\mathrm{x}=0 \mathrm{~mm}$ (figures $4(\mathrm{~b})$ and $4(\mathrm{e})$ ). This area is influenced by protons passing through both the thicker section of the $\mathrm{RC}$ and the higher-density region in the phantom. The third region is a low-dose region near $\mathrm{x}=0$ $\mathrm{mm}$ at a depth of $\mathrm{z}=90 \mathrm{~mm}$ (figures 4 (c) and $4(\mathrm{e})$ ) formed by protons passing through both the thinner section of the $\mathrm{RC}$ and the lower-density region in the phantom. Note that this region is located deeper than the target distal boundary. The RMPBA could not reproduce the dose in this region due to the disregard of irregular proton paths mentioned above while the SMC could.

Both the SMC and the RMPBA perfectly reproduced the depth-dose distribution in the region lacking lateral heterogeneity (figures $4(\mathrm{~d})$ and $4(\mathrm{f})$ ). The difference in calculation accuracy between the SMC and the RMPBA in figure 4(e) was caused by the difference in operation between the two algorithms; the SMC method tracks almost all proton paths while some paths in the RMPBA are missing.

\subsection{RANDO phantom}

Figures 5 and 6 describe the iso-dose distributions in the median sagittal plane and horizontal planes obtained using (a) measurements, (b) the SMC calculation, and (c) the RMPBA calculation. We ignored the $\mathrm{z}=6$ and $\mathrm{z}=7$ planes because almost no protons reached these levels. The number of measurement points irradiated with more than $10 \%$ of the normalization dose were $80,82,88,88$, and 41 for $\mathrm{z}=1$ through 5 . Apparent discrepancy between the target distal boundary and the dose distal boundary can be attributed to difference between the displayed depth and the measured depth by the detector with a cover thickness of $8 \mathrm{~mm}$ WEL. Since our interest is focused on difference between measurements and calculations, this is not a major issue here. In the figures, you will notice that both the SMC and the RMPBA reproduced the overall measured distribution in some accuracy. Yet there are some local differences shown in dose profiles of figure 7 : (a), (b), (c), (d), and (e) are lateral profiles at $\mathrm{z}=1-5$, 
and (f) is a distal profile at $\mathrm{y}=0 \mathrm{~mm}$. Figures $7(\mathrm{a})$, (b), and (c) show that both calculations reproduce measurements well with minor local differences. On contrary, we notice large discrepancy between measurements and calculations and difference between the SMC and the RMPBA in a part of figures $7(\mathrm{~d})$, (e), (f). The discrepancy between measurements and calculations is caused by the range uncertainty of the phantom and the large dose gradient in the distal fall-off part of the Bragg curve. The difference between the SMC and the RMPBA notably found in the figures (e) and (f) is caused by the fact that the RMPBA disregards dose contribution of protons passing through the irregular paths along the phantom and reaching the deep region and underestimates the dose in the deep region.

We also examined the dose error caused by misalignment of the $\mathrm{RC}$, aperture collimator, phantom, and detector. Figure 8 depicts three lateral dose profiles on the $\mathrm{z}$ $=4$ layer of the head phantom following three separate set-up procedures. The figure also contains the SMC and the RMPBA calculation results for the case of no set-up error. The $\mathrm{z}=4$ layer was selected since it includes a region with a large dose gradient in the lateral direction that is sensitive to set-up error. The measured dose error due to misalignment was a maximum of $9.2 \%$ p-p of the normalization dose. Such a large dose error can be attributed to an estimated setup error of $\pm 0.5 \mathrm{~mm}$ and the large dose gradient. The misalignment error is consistent with error of the alignment system using laser cross-hairs.

Figure 9 compares the dose-surface histograms (DSH) obtained from measurements, the SMC calculation, and the RMPBA calculation in each measurement plane. The SMC method is superior to the RMPBA, which underestimates the dose in deeper regions. The difference in calculation accuracy between the two algorithms arises from consideration or disregard of irregular proton paths in heterogeneous media. The dose underestimation observed in the $\mathrm{z}=5$ layer even by SMC is caused by uncertainty in the CT-value-to-range conversion and by the large low-density-region representing the oral cavity present in this layer. Since most protons in this region have a small residual range, the dose in this region is very sensitive to small uncertainties in the proton range.

\section{Discussion}

We evaluated the calculation accuracy using the $\gamma$-index method (Low 1998). This method simultaneously evaluates the dose-difference and the distance-toagreement quantitatively. We newly defined a signed-gamma-index $\left(\gamma_{ \pm}\right.$-index $)$at each measurement position to differentiate between overestimation and underestimation of the dose:

$\gamma_{ \pm}\left(\boldsymbol{r}_{m}\right)=\frac{D_{c}\left(\boldsymbol{r}_{m}\right)-D_{m}\left(\boldsymbol{r}_{m}\right)}{\left|D_{c}\left(\boldsymbol{r}_{m}\right)-D_{m}\left(\boldsymbol{r}_{m}\right)\right|} \times \min _{\boldsymbol{r}_{c}}\left[\sqrt{\frac{\left(D_{c}\left(\boldsymbol{r}_{c}\right)-D_{m}\left(\boldsymbol{r}_{m}\right)\right)^{2}}{D_{\text {tolerance }}^{2}}+\frac{\left(\boldsymbol{r}_{c}-\boldsymbol{r}_{m}\right)^{2}}{r_{\text {tolerance }}^{2}}}\right]$

where $D_{m}$ and $D_{c}$ represents the measured and calculated doses, $r_{c}$ and $r_{m}$ are the calculated and measured positions, and the parameters $D_{\text {tolerance }}$ and $r_{\text {tolerance }}$ are the 
tolerance error values of the dose and the distance. The acceptable error in proton therapy dose calculation for heterogeneous targets is not clearly defined at present. We followed the recommendations of Low (1998) for photon therapy, and used 3\% for $D_{\text {tolerance }}$ and $3 \mathrm{~mm}$ for $r_{\text {tolerance }}$ in this paper. We also defined the pass rate as the fraction of calculation points satisfying the condition of $-1<\gamma_{ \pm}<1$.

Figure 10 illustrates the $\gamma_{ \pm}$-index distributions on the median sagittal plane for (a) the SMC and (b) the RMPBA. Figure 11 contains stacked bar charts representing the $\gamma_{ \pm}$-index for (a) the SMC and (b) the RMPBA. We expressed the $\gamma_{ \pm}$-index scale using various intensities of red to highlight positive regions and blue for negative regions. The color intensity is proportional to the absolute value of the $\gamma_{ \pm}$-index. The pass rates in individual horizontal planes are also indicated under the z-index of the measurement planes. While calculation results obtained using the SMC method agreed well with the measurement results for layers $\mathrm{z}=1-4$, the pass rates for the RMPBA method fell bellow $90 \%$ in the $\mathrm{z}=4$ and 5 layers due to underestimation of the dose. Therefore, the SMC method is superior in the calculation accuracy to the RMPBA. Since the SMC method can accurately reproduce the measured dose distribution in complex media within a reasonable calculation time, it is capable of improving the accuracy of dose calculations in clinical situations.

\section{Conclusion}

We implemented the SMC method in the treatment planning system of the NCCHE in order to improve dose calculation accuracy in heterogeneous targets. The SMC method is easy to implement because it can use the same input data for PBAs. We verified the effectiveness of the SMC method by comparing the calculation results to the dose distributions measured at different depths in a heterogeneous slab phantom using a two-dimensional detector. We also measured the dose distributions at seven horizontal planes in an anthropomorphic phantom. For both of these cases, we found that the SMC method reproduced the measured dose distributions better than the RMPBA. In the slab phantom, we found that the RMPBA overestimated the dose in shallow regions and underestimated the dose in deep regions due to disregard of some proton paths in the heterogeneous region. The same tendency was also found for the RMPBA dose calculations for the anthropomorphic phantom. Since PBAs fundamentally have a risk of disregarding some proton paths, they may underestimate the dose in deep region for the case with large heterogeneity around the target.

We evaluated the treatment plan using the $\gamma_{ \pm}$-index analysis and found that the SMC method reproduced the measured dose distributions well within the accuracy tolerance of $3 \mathrm{~mm}$ and $3 \%$ in almost all regions. In addition, the calculation time required for the SMC method was about $30 \mathrm{~min}$ for a typical clinical case (target volume of $500 \mathrm{cc}$ ). The SMC method provides the higher calculation accuracy than RMPBA within a reasonable time, even for such a complex case. Since verification of the effectiveness of the SMC method is required for many clinical cases, we have begun 
a retrospective comparison between the $\mathrm{SMC}$ and PBA methods using previous proton therapy cases.

\section{Acknowledgments}

We appreciate SHI Accelerator Service Ltd, for their experimental support.

\section{References}

Agostinelli S et al 2003 GEANT4 - a simulation toolkit Nucl. Instrum. Methods. Phys. Res. A 506 250-303

Chen G T, Singh R P, Castro J R, Lyman J T and Quivey J M 1979 Treatment planning for heavy ion radiotherapy, Int. J. Radiat. Oncol. Biol. Phys. 5 1809-1819

Highland V L 1975 Some practical remarks on multiple scattering Nucl. Instrum. Methods 129 497-499

Highland V L 1979 Some practical remarks on multiple scattering Nucl. Instrum. Methods 161171

Hong L, Goitein M, Bucciolini M et al 1996 A pencil beam algorithm for proton dose calculations Phys. Med. Biol 41 1305-1330

Kanematsu N, Komori M, Yonai S and Ishizaki A 2009 Dynamic splitting of Gaussian pencil beams in heterogeneity-correction algorithms for radiotherapy with heavy charged particles Phys. Med. Biol. 54 2015-2027

Kohno R, Takada Y, Sakae T et al 2001 Range-modulated pencil beam algorithm for proton dose calculations Jpn. J. Appl. Phys 40 5187-5193

Kohno R, Sakae T, Takada Y et al 2002 Simplified Monte Carlo Dose Calculation for Therapeutic Proton Beams Jpn. J. Appl. Phys 41 L294-L297

Kohno R, Takada Y, Sakae T et al 2003 Experimental evaluation of validity of simplified Monte Carlo method in proton dose calculations Phys. Med. Biol 48 1277-1288

Kooy H M, Trofimov A, Engelsman M and Smith A R 2008 Treatment Planning Proton and Charged Particle Radiotherapy ed T F Delaney and H M Kooy (Philadelphia: Lippincott Williams \& Wilkins) pp70-107 chapter 8

Li J S, Shahine B, Fourkal E and Ma C M 2005 A particle track-repeating algorithm for proton beam dose calculation Phys. Med. Biol. 50 1001-1010

Low D A, Harms W B, Mutic S and Purdy J A 1998 A technique for the quantitative evaluation of dose distributions Med. Phys. 25 656-661

Nishio T, Kataoka S, Tachibana M et al 2006 Development of a simple control system for uniform proton dose distribution in a dual-ring double scattering method Phys. Med. Biol. 51 1249-1260

Paganetti H, Jiang H, Parodi K et al 2008 Clinical implementation of full Monte Carlo dose calculation in proton beam therapy Phys. Med. Biol 53 4825-4853

Petti P L 1992 Differential-pencil beam dose calculations for charged particles Med. Phys. 19 137-149

Schaffner B, Pedroni E and Lomax A 1999 Dose calculation models for proton treatment planning using a dynamic beam delivery system: an attempt to include density heterogeneity effects in the analytical dose calculation Phys. Med. Biol. 44 27-41

Sakae T et al 2000 Multi-layer Energy Filter for Realizing Conformal Irradiation in Charged Particle Therapy Med. Phys. 27 368-373

Spezi E, Angelini A L, Romani F and Ferri A 2005 Characterization of a 2D ion chamber array for the verifiation of radiotherapy treatments Phys. Med. Biol. 50 3361-3373

Szymanowski H, Mazal A, Nauraya C et al 2001 Experimental determination and verification of the parameters used in a proton pencil beam algorithm Med. Phys. 28 975-987

Tachikawa T, Sato T, Ogino T et al 1999 Proton beam therapy facilities of the National Cancer Center East Hospital Radiat. Indust. 84 48-53 
Takada Y, Himukai T, Takizawa K et al 2008 The basic study of a bi-material range compensator for improving dose uniformity for proton therapy Phys. Med. Biol. 53 5555-5569

Waters L 2002 MCNPX User's Manual version 2.4.0 Los Alamos National Laboratory LA-CP-02-408

Yepes P, Randeniya S, Taddei P and Newhauser W 2009 Monte Carlo fast dose calculator for proton radiotherapy: application to a voxelized geometry representing a patient with prostate cancer Phys. Med. Biol. 54 N21-N28 


\section{Figures:}

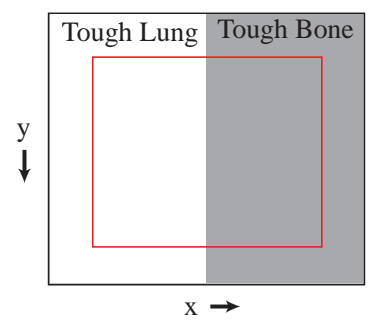

[Top View]

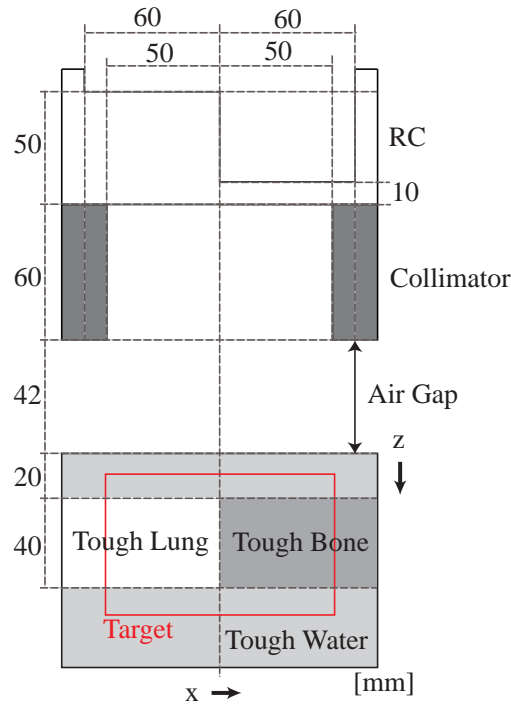

[Front View]

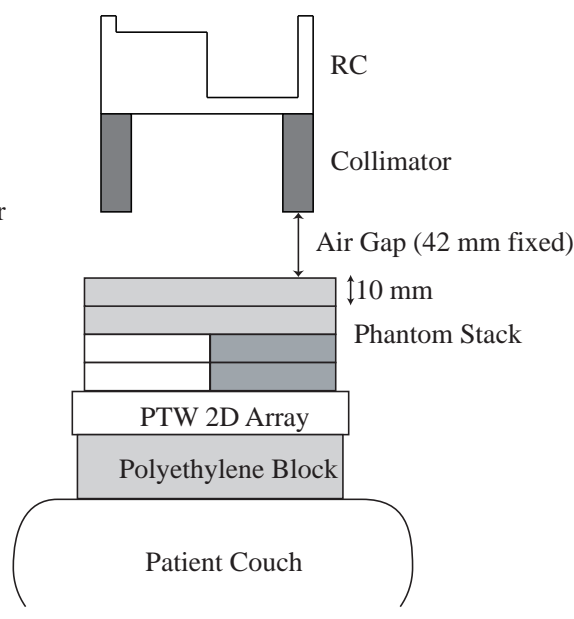

[Set Up (for z $=40 \mathrm{~mm})$ ]

Figure 1. Experimental arrangement of heterogeneous slab phantom, RC, and aperture collimator. The red line indicates the target region. 

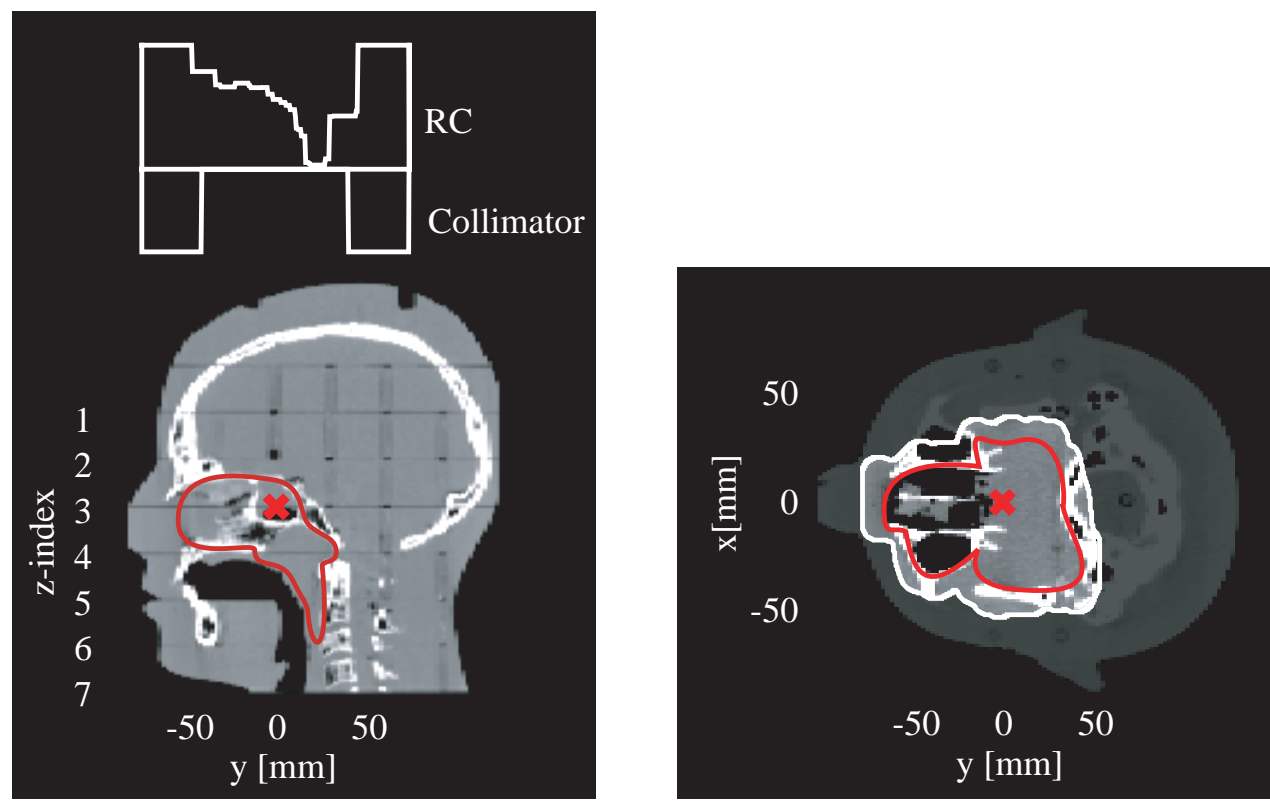

Figure 2. Median sagittal and median horizontal CT images of head portion from RANDO phantom. The iso-center (red cross mark), PTV (red line) and RC and collimator shape (white line) are also depicted. 
(a) Measurement

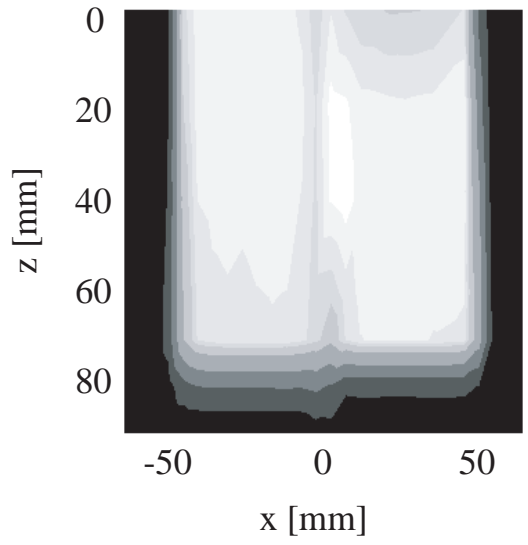

(b) SMC

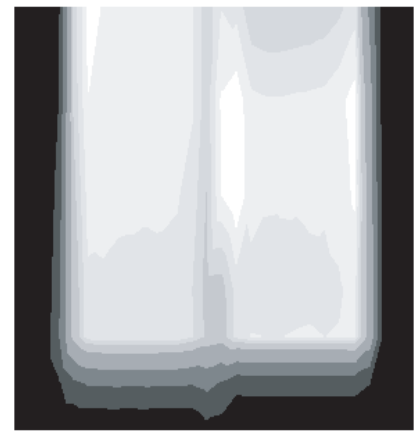

$-50$

0

50

$\mathrm{x}[\mathrm{mm}]$

(c) RMPBA

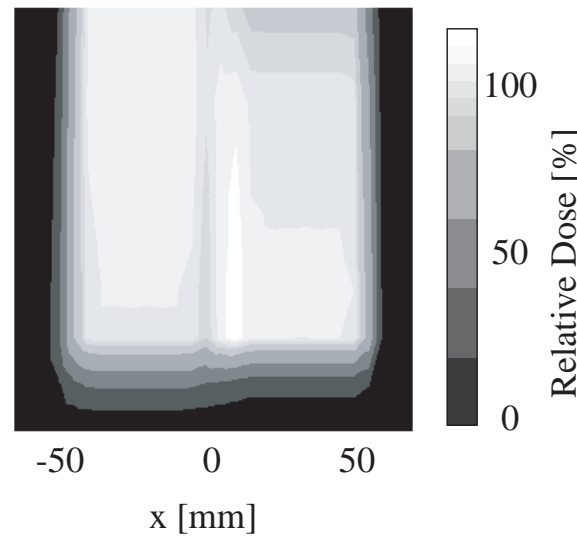

Figure 3. Iso-dose distributions in the heterogeneous slab phantom from (a) measurements, (b) the SMC, and (c) the RMPBA. The measurements were obtained at $\mathrm{z}=0 \mathrm{~mm}, 20 \mathrm{~mm}, 40 \mathrm{~mm}, 60 \mathrm{~mm}, 80 \mathrm{~mm}$, and $90 \mathrm{~mm}$. 
(a) Lateral dose profile at $\mathrm{z}=0 \mathrm{~mm}$

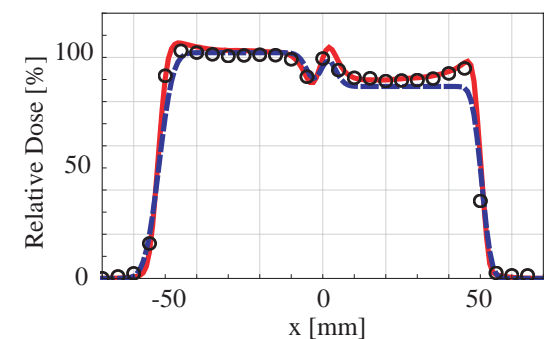

(d) Depth-dose profile at $\mathrm{x}=-20 \mathrm{~mm}$

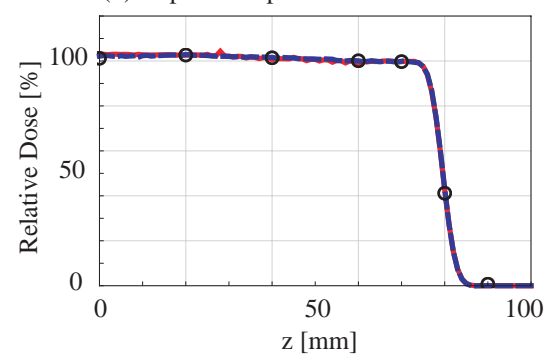

(b) Lateral dose profile at $\mathrm{z}=60 \mathrm{~mm}$

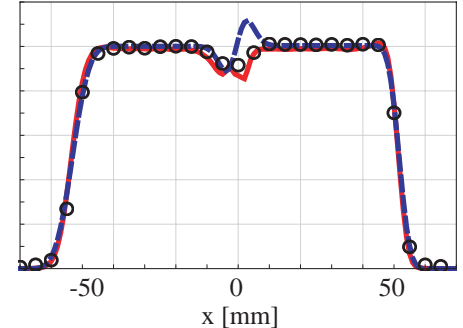

(e) Depth-dose profile at $\mathrm{x}=0 \mathrm{~mm}$

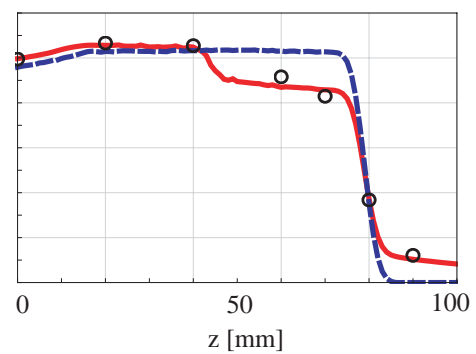

(c) Lateral dose profile at $\mathrm{z}=90 \mathrm{~mm}$

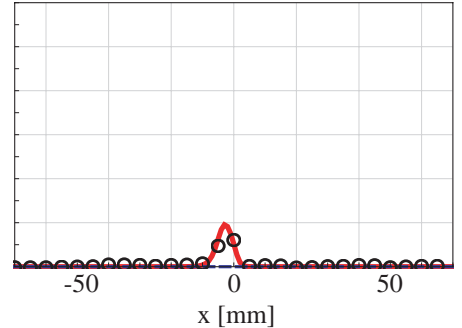

(f) Depth-dose profile at $\mathrm{x}=20 \mathrm{~mm}$

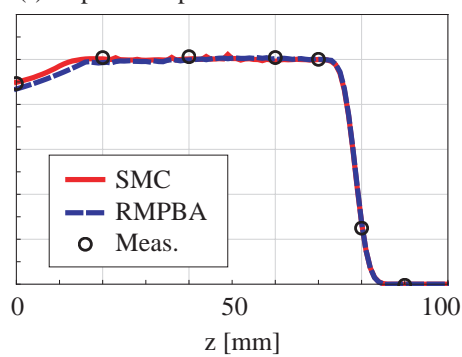

Figure 4. Dose profiles in the heterogeneous slab phantom; solid lines indicate the SMC prediction, dashed lines indicate the RMPBA prediction, and open circles represent measurements. Figures (a), (b), and (c) are lateral-dose profiles at $\mathrm{z}=0 \mathrm{~mm}$, $60 \mathrm{~mm}$, and $90 \mathrm{~mm}$. Figures (d), (e) and (f) are depth-dose profiles at y $=-20 \mathrm{~mm}$, $0 \mathrm{~mm}$, and $20 \mathrm{~mm}$. Since the estimated measurement error from three measurements in each set up are less than $1.0 \%$ p-p of the normalization dose, error bars are not displayed (smaller than circles). 


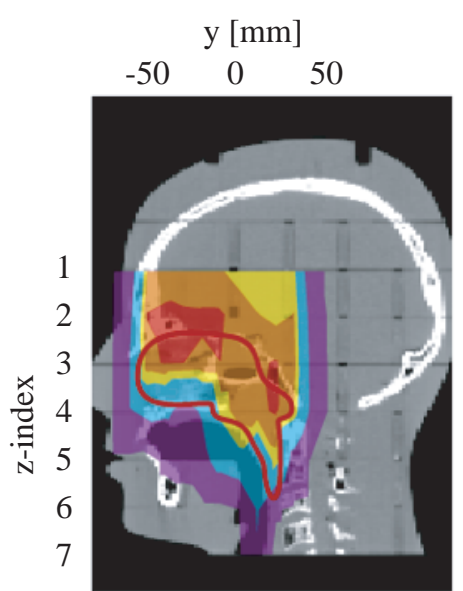

(a) Measurement

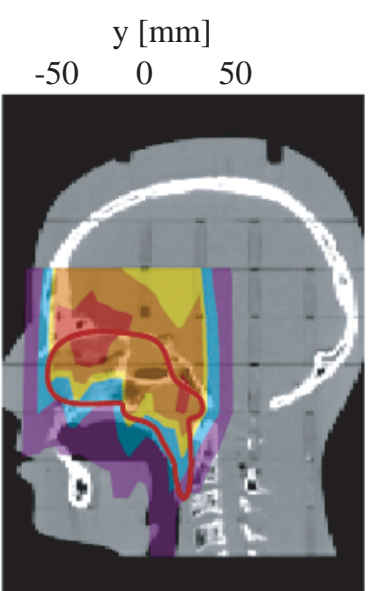

(b) SMC

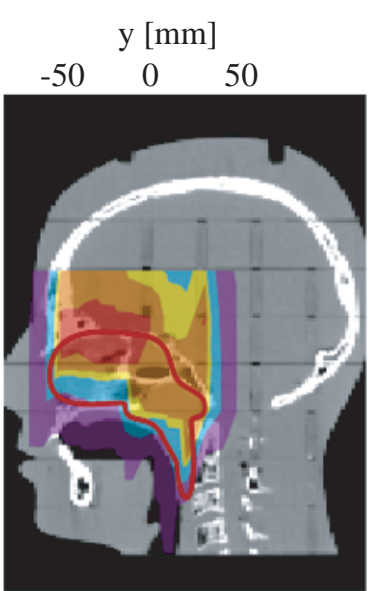

(c) RMPBA

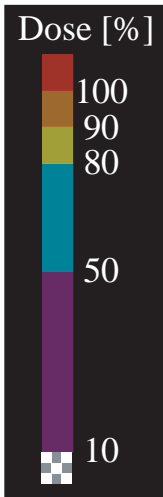

10

Figure 5. Median sagittal iso-dose distributions in the anthropomorphic phantom from (a) measurements, (b) the SMC, and (c) the RMPBA. 


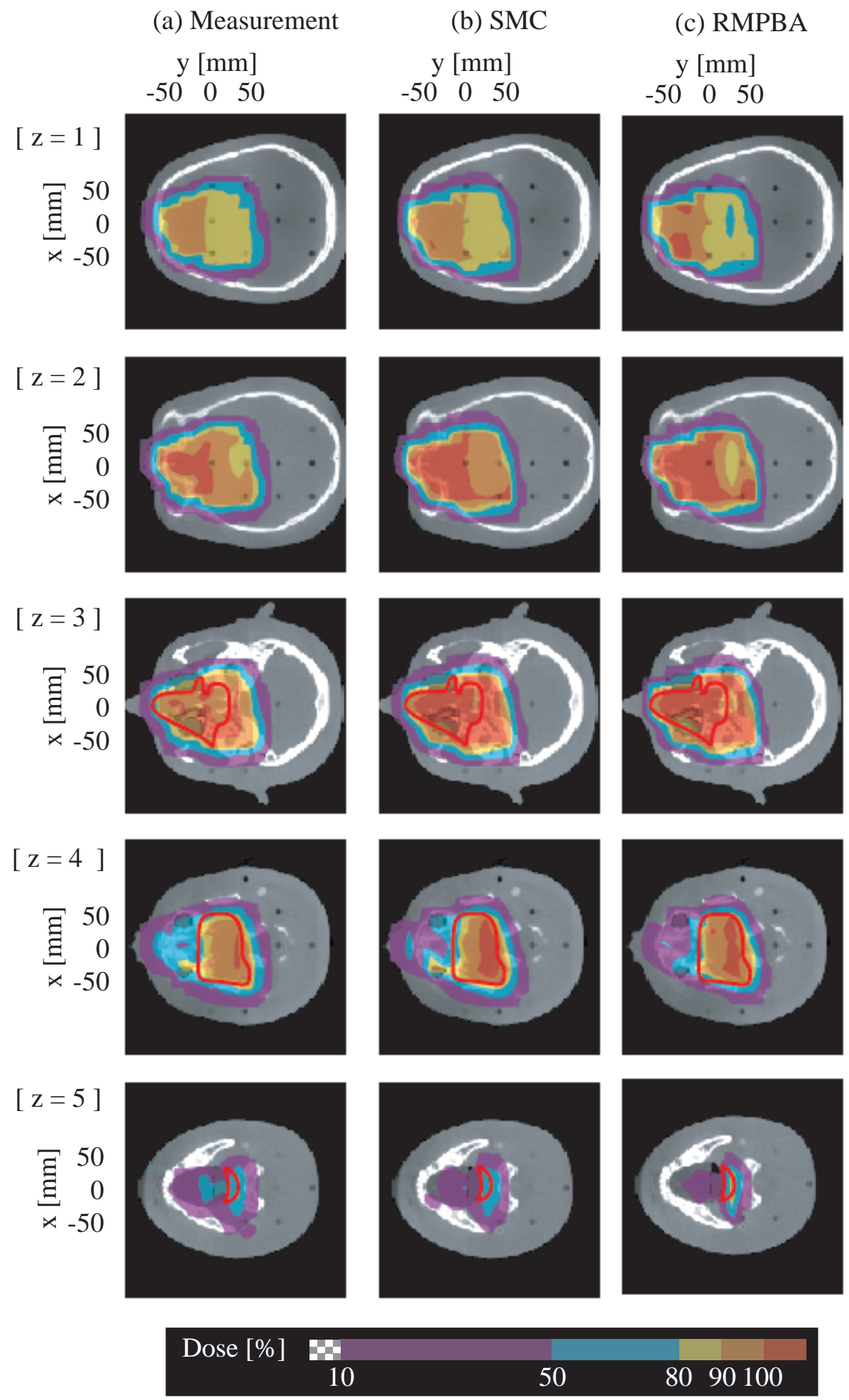

Figure 6. Horizontal iso-dose distributions in the anthropomorphic phantom. The red lines show the target region. 
(a) Lateral dose profile at $\mathrm{z}=1$

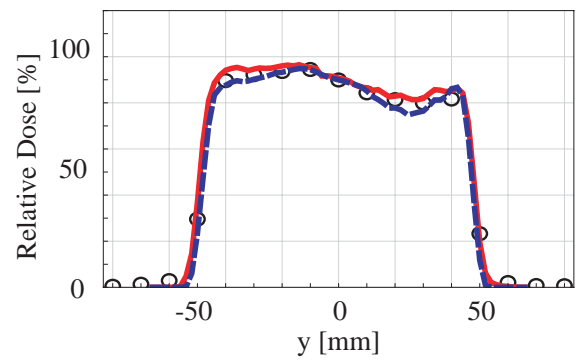

(d) Lateral dose profile at $\mathrm{z}=4$

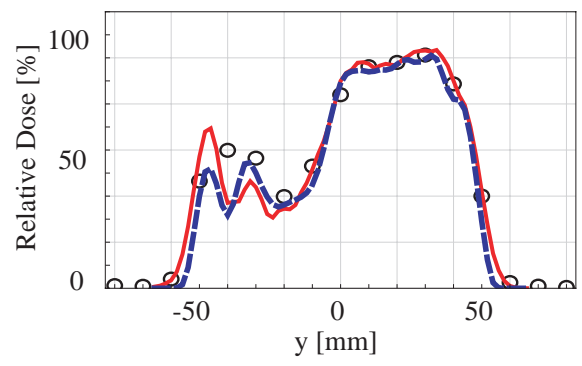

(b) Lateral dose profile at $\mathrm{z}=2$

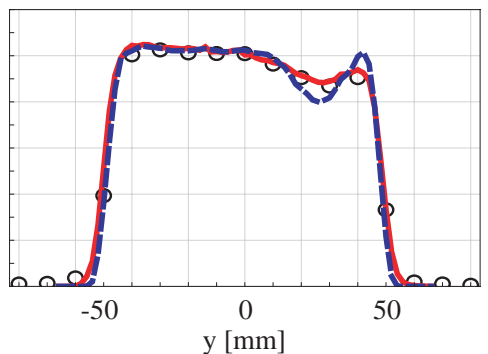

(e) Lateral dose profile at $\mathrm{z}=5$

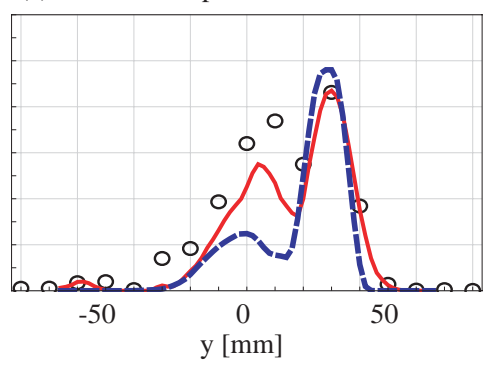

(c) Lateral dose profile at $\mathrm{z}=3$

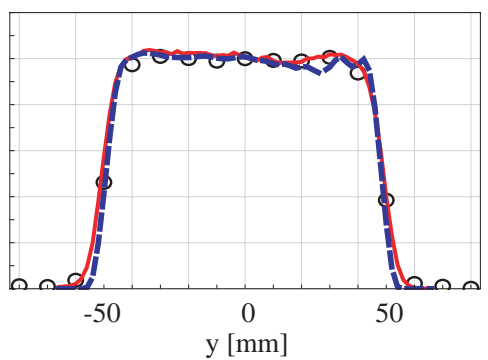

(f) Depth-dose profile at $\mathrm{y}=0 \mathrm{~mm}$

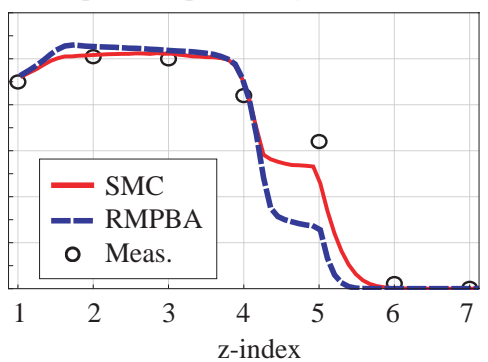

Figure 7. Lateral- and depth- dose profiles in the anthropomorphic phantom. The solid lines depict calculation results using the SMC, the dashed lines depict calculation results from the RMPBA, and the hollow circles represent measurements. Figures (a), (b), (c), (d) and (e) are lateral dose profiles on the $\mathrm{z}=1,2,3,4$, and 5 levels, and figure (f) is a depth-dose profile at $\mathrm{y}=0 \mathrm{~mm}$. Since the estimated measurement error from three measurements in each set up are less than $1.3 \%$ p-p of the normalization dose, error bars are not displayed (smaller than circles). 


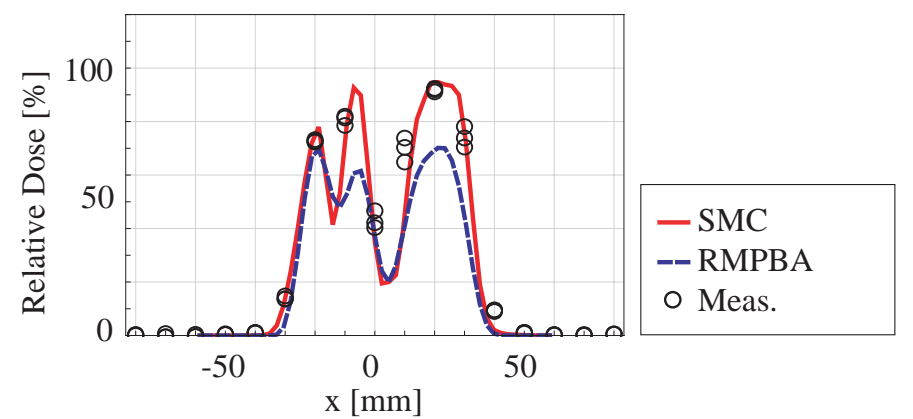

Figure 8. Effect of alignment error on dose distribution in the anthropomorphic phantom along the $\mathrm{x}$-axis at $\mathrm{y}=-20 \mathrm{~mm}$ on the $\mathrm{z}=4$ layer. The dose errors caused by set-up misalignment are large at $\mathrm{x}=10 \mathrm{~mm}$ and $\mathrm{x}=30 \mathrm{~mm}$ where the dose gradient is large. Since the estimated measurement error from times measurements in each set up are less than $0.7 \% \mathrm{p}-\mathrm{p}$ of the normalization dose, error bars are not displayed (smaller than circles). 

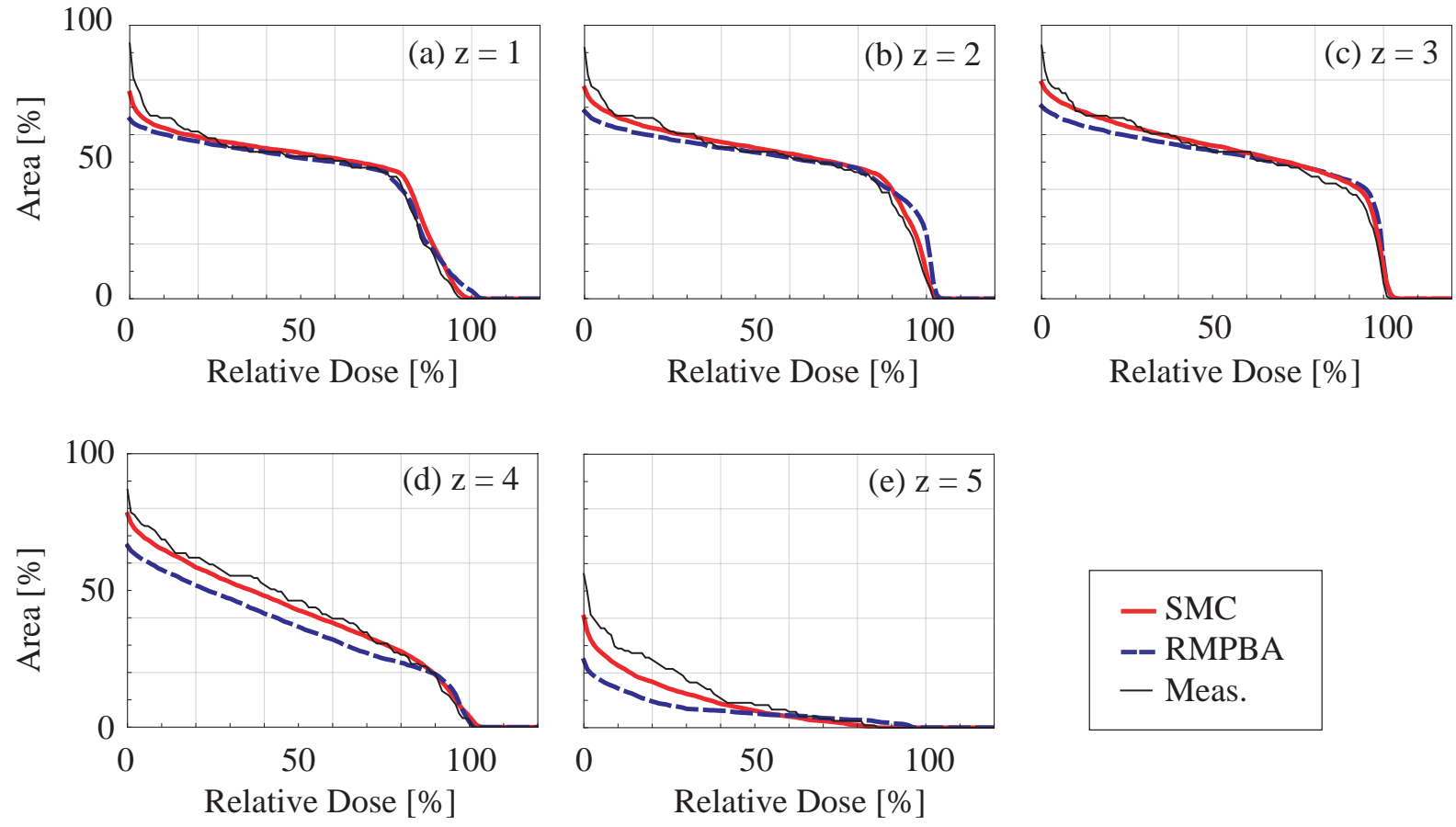

Figure 9. Comparison of dose-surface histograms in each plane from measurements, SMC calculation, and PBA calculation. The black solid lines depict the measurement data, the red solid lines depict the SMC results, and the blue dashed lines depict the RMPBA results. 


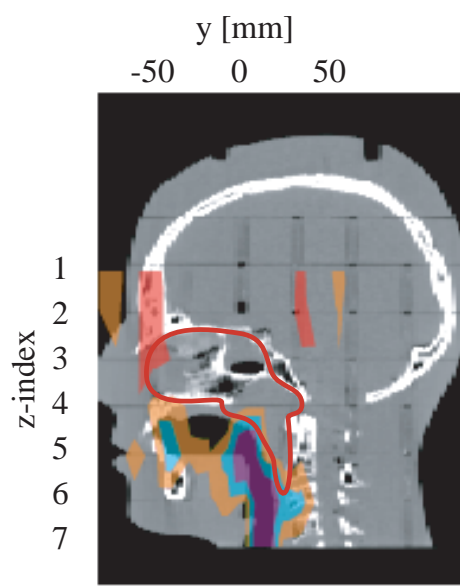

(a) SMC

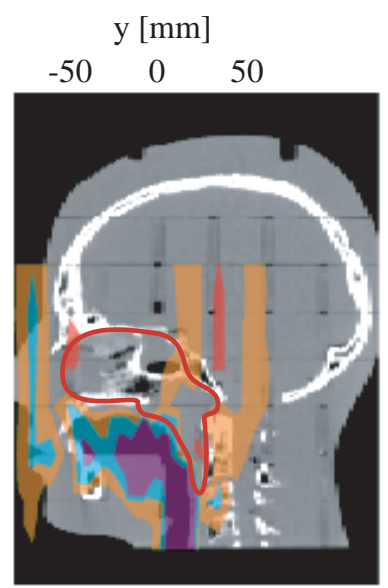

(b) RMPBA

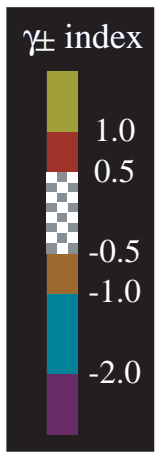

Figure 10. The $\gamma_{ \pm}$-index distributions in the median sagittal plane of the anthropomorphic phantom from (a) the SMC and (b) the RMPBA. The RMPBA exhibits a larger underestimated region than the SMC. 
(a) SMC

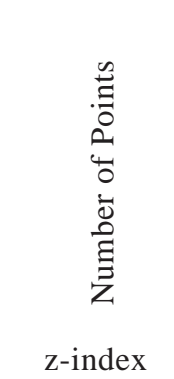

(Pass Rate [\%])

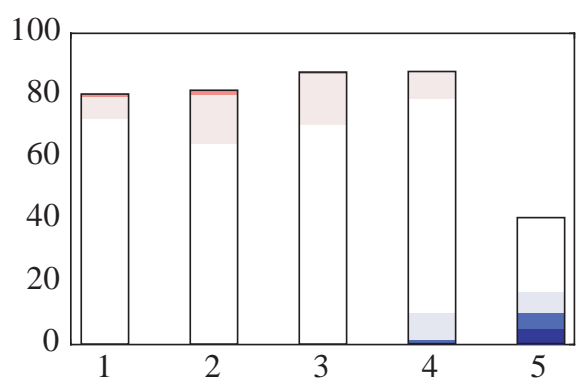

(99) (98) (99) (99) (76) (b) RMPBA

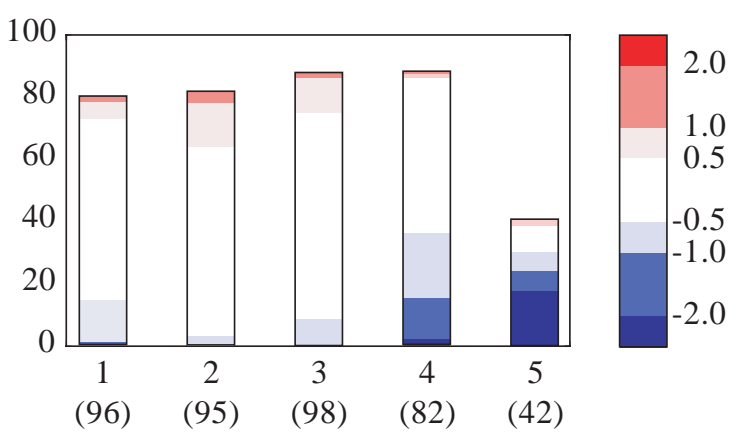

Figure 11. $\gamma_{ \pm}$-index histogram for (a) the SMC and (b) the RMPBA. The $\gamma_{ \pm}$-index in the stacked bar chart is shown in decreasing order from top to bottom. The number of points contained in a range of signed gamma index are indicated by the height of each bar. The pass rate is printed under the z-index. 